\title{
An Efficacious Text Summarization Process Using Triple Encoding-Decoding Mechanism
}

\author{
V.D.Ambeth Kumar a , R.SubhaShini ${ }^{\text {b }}$, K.Priyanka ${ }^{\text {b }}$ \\ ${ }^{a, b}$ Department of CSE, Panimalar Engineering College, Chennai
}

\begin{abstract}
As the big data and internet growing huge day by day, there is in need to overwhelm people by the information in large. These kind of issues makes researchers to create a technique that can generate automatic text. This research is very great interesting topic and the summarization of text is the process which undergoes finding important information within a document. Automatic text summarization is the process where a subset is created which represents the relevant content using the original text trained. So, text summarization is an intimidating challenge in Natural Language Processing because getting a good summary is a very big challenge as it is based on various aspects like metrics, nonredundancy, readability etc., At the outset, the text is trained into the system, next the trained text is fed into encoder decoder process where additional techniques are employed between the result in best coherent summary. The aim of automatic text summarization is to the produce finest and top quality text summary and our model also produces supreme quality summary.
\end{abstract}

Keywords-- big data, automatic text, text summary.

\section{Introduction}

This is an era where huge and huge amount of facts available on internet. It's a very main thing to give a well-developed technique to pick out important content very efficiently and rapidly. Enormous text materials are available on World Wide Web. So it is very strenuous process to find and search a document, and distilling relevant information from it. The automatic text summarization is necessary to solve the problem given before. Summarization is a thing which is highly necessary for the text summarization. So, it is important to know what is meant by a summary. A summary is nothing but a paragraph which is constructed by more texts, contains collection of sentences which conveys to give important needful information. The target of automatic text summarization is to present the original trained input text into a best version paragraph. The main and chief merit of using the summary is to reduce the time of reading 
Extractive and abstractive are types of summarization under text summarization. The extractive text summarization does a procedure of selection of main content which is sentences, words etc., from source text file that is concatenated to form a shorter summary. The abstractive text summarization does a procedure of understanding the subject and concept which is chief in the document and generates that concept in a clear form of summary.Informative and inductive are the two different kinds of text summarization where summarization of inductive does the representation of main text idea to the user. This type of summarization typically has as a length of $5-10 \%$ of text present in the main document. The summarization of informative system provides great information and typically has a length of $20-30 \%$ of text present in the main document.

\section{Literature Survey}

In the year of 2019, Md. MotiurRahman et al have proposed a system where the purpose is, from an input of long text an automatic summarization of text is generated by using a methodology of Multi-layered Attentional Peephole Convolution LSTM (Long Short-term Memory) (MAPCoL). The merit of the system is, it can generate a summary which is more precise [1].An Abstractive text summarization system is introduced by S Gehrmann et al in the year of 2018 where the system produces a content that is simple but also the selection of content which is accurate from the original text by using a methodology of data- efficient content selector and bottom-up attention. Here, the fluent summary of text is generated by using this approaches which results in compressing text ability [2]. In the year of 2018, K Al-Sabahi et al generated summaries with more and more novelty and high level of abstraction by proposing his system [18-31].

The summary of text is very much novel which is the main advantage here in this system where the system uses the methodology of end-to-end trainable bidirectional RNN model. A mechanism is also proposed that is bidirectional beam search mechanism with an algorithm [3]. AM Rush et al proposed a system which generates summary that must have each and every word present in input sentence trained in the year of 2015. The system uses the main methodology of local attention-based model which improves the quality as well as the inconsistency produced while the summary of text is generated. The demerit of the system is, while generating text summary there is no good improvement in the grammatical part [4]. The system of extractive document summarization is proposed where a neural sequence model for extractive document summary generation will allow the intuition for text visualization. The system is proposed by R Nallapati et al in the year of 2016 where a Summa RuNNer is presented by him that can give a better in performance [5].In the year of 2018, for conceptualization of extractive summary generation as a task of sentence ranking and for proposal of an algorithm of novel training, a system is proposed by S Narayan et al by using a methodology of sentence ranking and novel training algorithm. The CNN/DailyMail dataset is used here for the experiment where the system's algorithm is used for training the neural summarization model on the dataset used in this approach. The system is not perfect and gets confused in arranging sentences accordingly (i.e., ranking of sentences) is the demerit of this system [7].The abstractive text summarization system is proposed here to enhance semantic representation of text by $\mathrm{R}$ Nallapati in the year of 2016 [8]. The system uses a methodology of Attentional Encoder-Decoder Recurrent Neural Networks and also several novel models are 
proposed which will provide some of the problems happened in the summarization. He also proposes a new dataset which will have the summaries of multi-sentence and the disadvantage of this system is, long text semantic representation is very difficult.

\section{System Architecture}

The diagrammatic representation of the text summarization process is shown below. The system is trained with input text which is employed to triple encoding. The result of the triple encoding and the key information, contextual information from the triple encoding result is fed into the process decoding. Thus, the decoder outputs the resultant automatic text summary.

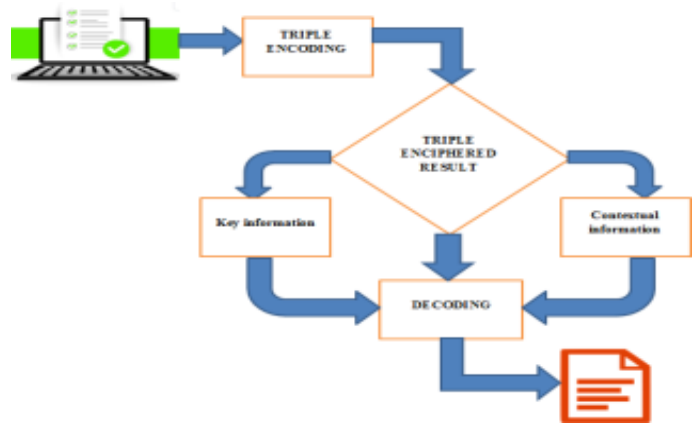

Figure 1. Text summarization process

\section{Data Flow Diagrams}

\section{1 level 0}

The system is trained with the input source text where this trained text is fed into the part of triple encoding and decoding and the target summary result is obtained.

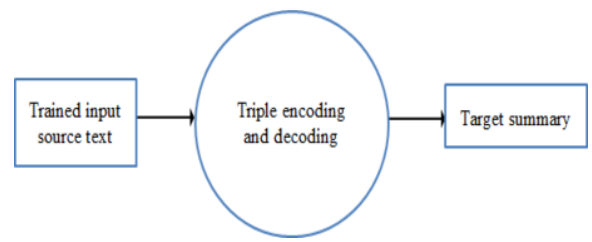

\subsection{Level1}

Figure 2. Level 0 DFD

The trained input text is given into triple encoding to produce first and second encipher result. The result of first and second encipher is collected into double enciphered result which is fed into third encipher process. The third encipher is fed into decoding process to result in target summary. 


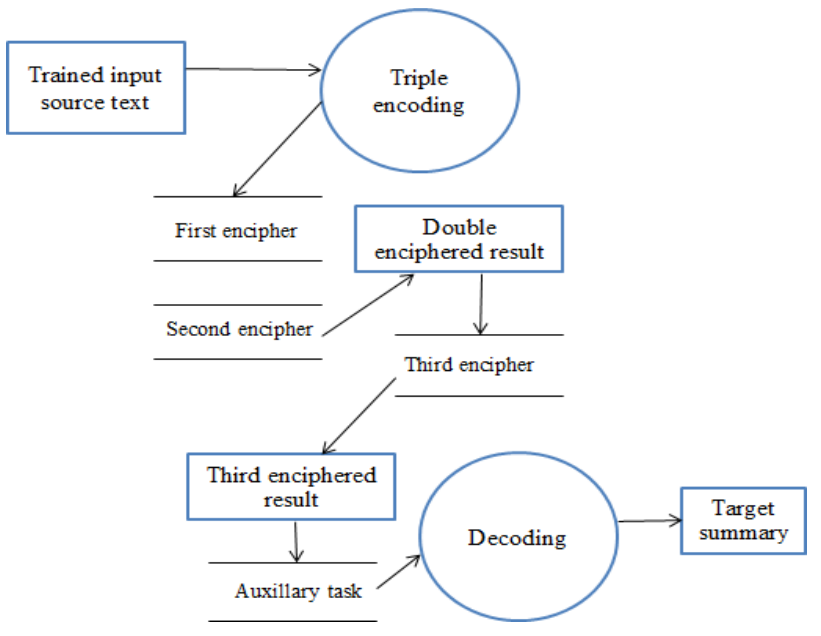

Figure 3. Level 1 DFD Diagram

\section{Conclusion}

Thus, the automatic text summarization process produces finest and top quality text summary and also produces supreme quality summary. The text is trained into the system, next the trained text is fed into encoder-decoder process where additional techniques are employed between, to result in generation of best summary.

\section{References}

[1] Rahman, M., Siddiqui, F.H..An Optimized Abstractive Text Summarization Model Using Peephole Convolutional LSTM. mdpi-Symmetry,2019.

[2] Gehrmann, S., Deng, Y., Rush AM..Bottom-up abstractive summarization. Proceedings of the 2018 Conference on Empirical Methods in Natural Language Processing, Association for Computational Linguistics,2018.

[3] Al-Sabahi, K., Zuping, Z., Kang, Y..Bidirectional Attentional Encoder-Decoder Model and Bidirectional Beam Search for Abstractive Summarization. Computation and Language (cs.CL), 2018.

[4] Rush, A.M., Chopra, S., Weston, J..A neural attention model for abstractive sentence summarization. Proceedings of the 2015 Conference on Empirical Methods in Natural Language Processing,2015.

[5] Nallapati, R., Zhai, F., Zhou, B..Submariner: A recurrent neural network based sequence model for extractive summarization of documents. The Thirty-First AAAI Conference on Artificial Intelligence, 2016.

[6] Zhou, Q., Yang, N., Wei, F., Huang, S., Zhou, M..Neural document summarization by jointly learning to score and select sentences. The 56th Annual Meeting of the Association for Computational Linguistics, 2018.

[7] Narayan, S., Cohen, S.B., Lapata, M.: Ranking sentences for extractive summarization with reinforcement learning. the 16th Annual Conference of the North American Chapter of the Association for Computational Linguistics,2018.

[8] Nallapati et.al, Abstractive text summarization using sequence- to-sequence rnns and beyond. The SIGNLL Conference on Computational Natural Language Learning (CoNLL), 2016.

[9] Cheng, J., Lapata, M..Neural summarization by extracting sentences and words. the 54th Annual Meeting of the Association for Computational Linguistics, 2016.

[10] Jen-TzungChien and Yuan-Chu Ku.Bayesian Recurrent Neural Network for Language Modeling. IEEE Transactions on Neural Networks and Learning Systems Volume: 27, Issue: 2,2016.

[11] Chopra, S.et.al.Abstractive sentence summarization with attentive recurrent neural networks. Proceedings of the conference of NAACL-HLT, Association for Computational Linguistics, 2016. 
[12] Deepanagalavi, Hanumanthappa, M., Ravikumar, K..An Improved Attention Layer assisted Recurrent Convolutional Neural Network Model for Abstractive Text Summarization. INFOCOMP Journal of Computer Science, 2019.

[13] Qingyu Zhou, Nan Yang, Furu Wei, Ming Zhou.Selective Encoding for Abstractive Sentence Summarization. Asia-Pacific Web (APWeb) and Web-Age Information Management (WAIM), 2017.

[14] QianGuo, Jifeng Huang, NaixueXiong, Pan Wang: MS-Pointer Network.Abstractive Text Summary Based on Multi-Head Self-Attention. IEEE Access, Journal Article, Publisher: IEEE, Vol. 7, 2019.

[15] Song, S., Huang, H., \&Ruan, T., "Abstractive text summarization using LSTM-CNN based deep learning”, Multimedia Tools and Applications 78, Springer, 857-875,2019.

[16] Bing, L., Li, P., Liao, Y., Lam, W., Guo, W., “Abstractive multi-document summarization via phrase selection and merging”, Association for Computational Linguistics (ACL),2015.

[17] KaichunYao,LiboZhang,DaweiDu,TiejianLuo,LiliTao,YanjunW

[18] S.Hema Kumar, J.UdayKiran, V.D.AKumar, G.Saranya, Ramalakshmi V, "Effective Online Medical Appointment System”, International Journal of Scientific \& Technology Research, Volume 8, Issue 09, September 2019, Pages 803 - 805.

[19] V.D.Ambeth Kumar, Dr.M.Ramakrishnan, V.D.Ashok Kumar and Dr.S.Malathi (2015) "Performance Improvement using an Automation System for Recognition of Multiple Parametric Features based on Human Footprint" for the International Journal of kuwait journal of science \& engineering, Vol 42, No 1 (2015), pp:109-132.

[20] V.D.Ambeth Kumar, V.D.Ashok Kumar, S.Malathi, K.Vengatesan, M.Ramakrishnan, "Facial Recognition System For Suspect Identification Using A Surveillance Camera", Pattern Recognition and Image Analysis (Springer), Volume 28, Issue 3, pp 410-420, $2018 . \quad$ (DOI: $10.1134 / \mathrm{S} 1054661818030136)$

[21] V.D.Ambeth Kumar and M.Ramakrishan. Employment Of Footprint Recognition System. in the month of December for Indian Journal of Computer Science and Engineering (IJCSE) Vol. 3 No.6 Dec 2013

[22] Ramya,T.,Dr.Malathi,S.,ratheeksha,G.R. and Dr.V.D.Ambeth Kumar (2014). Personalized authentication procedure for restricted web service access in mobile phones. Applications of Digital Information and Web Technologies (ICADIWT), 2014, Page(s):69 - 74, Bangalore, India (ISBN:978-14799-2258-1)

[23] Ambeth Kumar.V.D et.al.Novel Wireless Sensing System For The Welfare Of Sewer Laborers. Healthcare Technology Letters (IET) Volume 5, Issue 4, p. $107-112,2018$

[24] Ambeth Kumar.V.D, G.Saranya , D.Elangovan, V.RahulChiranjeevi, V.D.AshokKumar.IOT Based Smart Museum Using Wearable Device.Lecture Notes in Networks and Systems, Vol.55, pp: 33-42, 2018

[25] Ambeth Kumar.V.D and Dr.M.Ramakrishan .Footprint Based Recognition System. in the month of April for the International Journal Communication in Computer and Information System, Volume 147, Part 3, 358-367, DOI: 10.1007/978-3-642-20573-6 63, April 2011

[26] Ambeth Kumar.V.D (2017) .Efficient Routing for Low Rate Wireless Network a Novel Approach. International Journal of Image Mining, Vol. 2, Nos. 3/4, 2017, 2017

[27] V.D.Ambeth Kumar, V.D.Ashok Kumar, Dr.S.Malathi and P.Jagaeedesh, (2014) "Intruder Identification using Footprint Recognition with PCA and SVM Classifiers" for the International Journal of Advanced Materials Research Vols.1345, PP 984-985 (2014) pp 1345-1349. [DOI:10.4028/www.scientific.net/AMR.984-985.1345]

[28] Ambeth Kumar.V.D, G.Gokul, S.Malathi, K.Vengatesan, D.Elangovan, B.Chitra.Implementation Of The Pulse Rhythemic Rate For The Efficient Diagonising Of The Heart Beat.Healthcare Technology Letters (IET) 2019 Apr 17;6(2):48-52.

[29] S.V. Ruphitha et.al, Management of Major Postpartum Haemorrhage by using Zigbee protocol - A Review. 2021 6th International Conference on Inventive Computation Technologies (ICICT) (DOI: 10.1109/ICICT50816.2021.9358757)

[30] Ambeth Kumar.V.D, Dr.M.Ramakrishnan, V.D.Ashok Kumar and Dr.S.Malathi (2015) "Performance Improvement using an Automation System for Recognition of Multiple Parametric Features based on Human Footprint" kuwait journal of science, Vol 42, No 1 (2015), pp:109-132.

[31] M.Ramakrishan et.al..Footprint Based Recognition System. in the month of April for the International Journal Communication in Computer and Information System (CCIS) Journal (Springer) Volume 147, Part 3, 358-367, DOI: 10.1007/978-3-642-20573-6 63, April 2011

[32] Indhumathi.M et.al .Healthcare Management of Major Cardiovascular Disease-A review. 2021 6th International Conference on Inventive Computation Technologies (ICICT), (DOI: 10.1109/ICICT5081.

[33] R. Subha Shini et.al., " Recurrent Neural Network based Text Summarization Techniques by Word Sequence Generation”, IEEE International Conference on Inventive Computation Technologies (ICICT), 2021, DOI: 10.1109/ICICT50816.2021.9358764 